ARTICLE

https://doi.org/10.1038/s41467-019-11178-w

\title{
Patient-derived pancreas-on-a-chip to model cystic fibrosis-related disorders
}

Kyu Shik Mun¹, Kavisha Arora1, Yunjie Huang ${ }^{1}$, Fanmuyi Yang ${ }^{1}$, Sunitha Yarlagadda', Yashaswini Ramananda ${ }^{1,2}$, Maisam Abu-El-Haija ${ }^{3,4}$, Joseph J. Palermo ${ }^{3,4}$, Balamurugan N. Appakalai ${ }^{5}$, Jaimie D. Nathan ${ }^{6} \&$ Anjaparavanda P. Naren ${ }^{1}$

Cystic fibrosis (CF) is a genetic disorder caused by defective CF Transmembrane Conductance Regulator (CFTR) function. Insulin producing pancreatic islets are located in close proximity to the pancreatic duct and there is a possibility of impaired cell-cell signaling between pancreatic ductal epithelial cells (PDECs) and islet cells as causative in CF. To study this possibility, we present an in vitro co-culturing system, pancreas-on-a-chip. Furthermore, we present an efficient method to micro dissect patient-derived human pancreatic ducts from pancreatic remnant cell pellets, followed by the isolation of PDECs. Here we show that defective CFTR function in PDECs directly reduced insulin secretion in islet cells significantly. This uniquely developed pancreatic function monitoring tool will help to study CF-related disorders in vitro, as a system to monitor cell-cell functional interaction of PDECs and pancreatic islets, characterize appropriate therapeutic measures and further our understanding of pancreatic function.

\footnotetext{
${ }^{1}$ Department of Pediatrics, Division of Pulmonary Medicine, Cincinnati Children's Hospital Medical Center, Cincinnati, OH 45229, USA. ${ }^{2}$ Department of Medical Biotechnology, University of Illinois, Rockford, IL 61107, USA. ${ }^{3}$ Division of Pediatric Gastroenterology, Hepatology and Nutrition, Cincinnati Children's Hospital Medical Center, Cincinnati, OH 45229, USA. ${ }^{4}$ Department of Pediatrics, University of Cincinnati, Cincinnati, OH 45229, USA. ${ }^{5}$ Department of Surgery, Center for Cellular Transplantation, Cardiovascular Innovation Institute, University of Louisville, Louisville, KY 40222, USA. ${ }^{6}$ Division of Pediatric General and Thoracic Surgery, Cincinnati Children's Hospital Medical Center, Cincinnati, OH 45229, USA. Correspondence and requests for materials should be addressed to J.D.N. (email: jaimie.nathan@cchmc.org) or to A.P.N. (email: anaren@cchmc.org)
} 
T he cystic fibrosis transmembrane conductance regulator (CFTR) protein is located on the apical membrane of epithelial cells in multiple organs, including lung, sweat gland, gastrointestinal tract, and pancreas, and its dysfunction is responsible for the clinical manifestations of cystic fibrosis $(\mathrm{CF})^{1-4}$. CFTR is a cyclic AMP (cAMP)-dependent chloride and bicarbonate transport channel and plays an important role in maintaining salt and water balance on the epithelial surface. Defective CFTR channel function lowers the water content in the lumen, which leads to the development of a thick and viscous mucus on the epithelial surfaces in CF-affected organs ${ }^{3}$. To date, more than 2000 CFTR mutations have been identified since the first discovery of $\mathrm{CF}$ in $1938^{5}$. CFTR mutations are classified into six categories according to the primary molecular defect of the CFTR protein: synthesis (class I), trafficking process (II), gating (III), conductance (IV), mRNA stability (V), and CFTR stability (VI) ${ }^{6}$.

CF-related diabetes (CFRD) is a frequent and deadly complication in CF. A patient with CF has an increasing risk of developing diabetes with age of $5 \%$ per year, reaching $50 \%$ by age $40 \mathrm{~s}^{7,8}$. CFRD affects $2 \%$ of children, $19 \%$ of adolescents, and as high as 50\% of adults ${ }^{7}$. Glucose imbalance due to CFRD has been correlated with increased morbidity and mortality in patients with CF. This calls for a need to develop approaches to study CFRD and identify therapeutic measures to potentially manage disordered glucose metabolism in CFRD. CFRD is complex as it exhibits the features of both the lack of insulin typical of type 1 diabetes (T1D) and the insulin resistance typical of type 2 diabetes (T2D) ${ }^{9}$. Whether a lack of CFTR function in CF patients directly manifests into CFRD remains unclear. Patients with CFRD show more severe side effects with significant loss of lung function and imbalanced nutrition than $\mathrm{CF}$ patients without diabetes ${ }^{10}$. CFTR is highly expressed in the pancreatic ductal epithelial cells (PDECs) ${ }^{1,11-13}$, which are located in close proximity to pancreatic islets ${ }^{14}$; however,. the functional relationship between these two cell types in CFRD remains unclear. To investigate the role of CFTR in CFRD and functional correlation between PDECs and islet cells, we isolated PDECs and pancreatic islets from pancreatitis patients, who underwent total pancreatectomy with islet autotransplantation (TPIAT) ${ }^{15}$. We cultured these cell types in a microfluidic device to develop pancreas-on-achip, an in vitro model system to mimic the functional interface between PDECs and pancreatic islets. The pancreas-on-a-chip allows us to monitor cell-cell functional interaction directly and efficiently using only a small number of patient-derived cells. The microfluidic device was developed in 1979 as a miniature gas chromatograph ${ }^{16}$ and it has been exponentially innovated in functionality and design. In recent decades, microfluidics have been used as an in vitro model system for cell culture ${ }^{17-19}$, because of its high reproducibility, ability to mimic function and structure of organs, and some unique applications such as realtime $\mathrm{PCR}^{20}$, single-cell western blot ${ }^{21}$, wearable sensor ${ }^{22}$, and organ-on-a-chip ${ }^{23,24}$. Pancreas-on-a-chip will facilitate elucidating the mechanism of cross-talk between PDEC and islet cells, which is key to understanding the relationship between $\mathrm{CF}$ and diabetes. Classified mutations in the CFTR gene may show variable pathological processes in the development of CFRD.

Here, we have successfully co-cultured patient-derived PDECs and islet cells in the same chip and observed that attenuating CFTR function in PDECs reduces insulin secretion in islet cells by $54 \%$. This pancreas-on-a-chip is an innovative approach of developing personalized medicine to address heterogeneity in CFRDs.

\section{Results}

Isolation of patient-derived PDECs and pancreatic islets. Pancreatitis patients who have a debilitating course of acute recurrent or chronic pancreatitis may undergo TPIAT) to relieve their pain and incapacitation, as shown in Fig. 1a. During TPIAT, the pancreas is resected and digested to isolate pancreatic islets. The islets are infused into the liver through the portal vein to engraft within the hepatic sinusoids and maintain their endocrine function. The pancreatic remnant cell pellet was obtained after islet cell isolation (Fig. 1b) and shown to contain pancreatic islets, acinar cells, and PDECs) (Fig. 1c). Pancreatic islets in the pancreatic remnant cell pellet were visualized by adding dithizone solution, which turns the color of islets to red (Fig. 1d) ${ }^{25}$. The clustered red-colored pancreatic islets were manually isolated and cultured in vitro (Fig. 1g). The diameter of the isolated pancreatic islets ranged from 50 to $300 \mu \mathrm{m}$.

From the pancreatic remnant cell pellet, we successfully isolated the pancreatic duct with diameter ranging from 90 to $1000 \mu \mathrm{m}$ by microdissection under a stereo microscope (Fig. 1e). Hematoxylin and eosin (H\&E) staining of the pancreatic duct demonstrated that the predominant cells, PDECs, were surrounded by collagen and connective tissue (Fig. 1f). Pancreatic ducts were enzymatically digested to separate the cells (Supplementary Fig. 1). Isolated pancreatic ductal cells were subsequently embedded in Matrigel and clustered into small spherical structures after isolation (day 0 ) and formed organoid structures at day 1, with a luminal fluid-filled area in the center (Fig. 1h). The organoids grew into larger spheres with a diameter of approximately $400 \mu \mathrm{m}$ at day 6 from isolation. Growth of organoids could exceed $3 \mathrm{~mm}$ in diameter. Organoids in Matrigel-formed duct-like structures when they contacted the surface of the substrate and migrated out forming a monolayer (Fig. 1i). Although the mechanism is unclear, we have consistently observed this phenomenon (Supplementary Fig. 2). To assist forming a monolayer of PDECs, the organoids were hand-picked manually and transferred to a fresh culture dish or trans-well membrane following breaking down of the Matrigel. During hand-picking, the organoids were separated from the Matrigel and collapsed by pipetting. Collapsed organoids attached to the surface, and PDECs started migrating out from organoids forming a monolayer (Supplementary Movie 1). From the monolayer of PDECs, single cells were harvested and embedded into fresh Matrigel. The PDECs re-formed organoid structures and grew over time (Fig. 1j). Furthermore, we have succeeded in freezing and reviving patient-derived PDECs using transformation of the organoid-monolayer structure (Fig. 1k). Hence, we have standardized the protocol to isolate, culture, and expand patient-derived ductal epithelial cells and pancreatic islets to provide a platform for the development of personalized medicine in pancreas-related disorders such as CFRD.

Characterization of PDECs. PDECs are one of the most abundant cell type present in the pancreas. We intended to confirm whether the isolated cells from the pancreatic remnant cell pellet were indeed PDECs. Isolated pancreatic ductal organoids (Fig. 2a) were first examined using standard morphological H\&E staining (Fig. 2d). The images demonstrated that ductal epithelial cells are located at the edge of the organoid, with a central luminal area. Immunofluorescence images showed pancreatic ductal organoids expressing epithelial cell biomarkers, cytokeratin 19 (KRT 19) (Fig. 2b), E-cadherin (Fig. 2c), sodium transport channel (ENaC) (Fig. 2e), and tight junction protein (ZO-1) (Fig. 2f). We cultured PDEC-derived monolayer (Fig. 2g) from the ductal organoids and detected positive immunofluorescent signals corresponding to epithelial cell biomarkers, ZO-1 (Fig. 2h), F-actin, and KRT 19 (Fig. 2i). Paraffin-sectioned H\&E images demonstrated a monolayer of PDECs (Fig. 2j) obtained from organoids. From the PDECs on the trans-well membrane, we observed CFTR located 


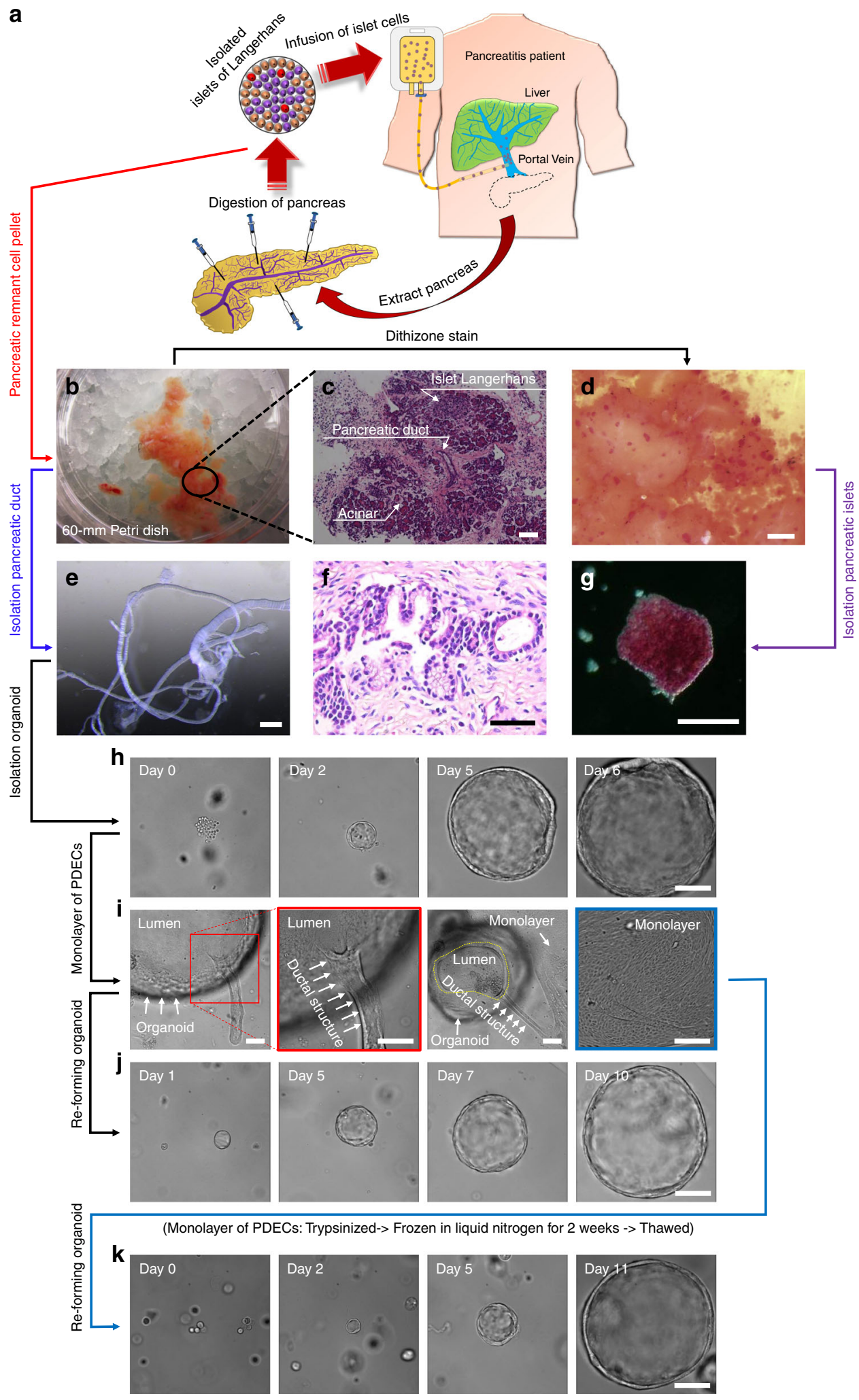

on the apical membrane of the ductal epithelial cells (Fig. 2k). Polarized monolayer of PDECs on a porous membrane in a pancreas-on-a-chip was examined with immunofluorescence image of ZO-1 and measurement of transepithelial electrical resistance using epithelial volt-ohm meter (Supplementary Fig. 3).

We performed RNA-sequencing in these organoids and verified that the organoids were of human PDECs in origin (Fig. 2l). Epithelial cell markers, cytokeratin family proteins (KRT
7, KRT 8, and KRT 19), CFTR, and E-cadherin were highly expressed in the organoids; however, blood cell marker (CDH 5), pancreatic acinar cell biomarkers, CPA1, GP2, and amylase (AMY2A), and pancreatic endocrine markers, insulin, glucagon, and somatostatin were not expressed. Hence, we sufficiently validated that the cell population processed from the pancreatic remnant cell pellet is purely ductal epithelium in origin, which is critical to developing our proposed model system to investigate 
Fig. 1 Isolation of patient-derived pancreatic ductal epithelium and islet cells. a Schematic representation of the total pancreatectomy with islet autotransplantation (TPIAT) procedure for pancreatitis patient. $\mathbf{b}$ Digested pancreatic remnant cell pellets were obtained following isolation of islet cells for infusion. c Hematoxylin and eosin (H\&E stain) image demonstrates that the remnant cell pellet contains pancreatic islets, ductal epithelial cells, and acinar cells. $\mathbf{d}$ Pancreatic islets were identified by adding dithizone solution, where the color turned to red, and $\mathbf{g}$ isolated by manual pipetting. e Pancreatic ductal tissues were isolated by microdissection from the pancreatic remnant cell pellet following TPIAT. $\mathbf{f} H \& E$ staining showed that pancreatic ductal epithelial cells were surrounded by collagen and connective tissue. $\mathbf{h}$ Pancreatic ductal epithelial cells (PDECs) were isolated from the ductal tissue and embedded in Matrigel matrix. PDECs grew into large spheres over time. i PDECs extend from the isolated organoid to form a monolayer on the surface of the substrate. j Ductal epithelial cell monolayers were re-formed into an organoid structure in the Matrigel and grew into a large sphere again. $\mathbf{k}$ Revived ductal epithelial cells following cryopreservation were embedded in the Matrigel and formed into large spheres over time. Scale bars: $50 \mu \mathrm{m}$ (f), $100 \mu \mathrm{m}$ (c, $\mathbf{g}-\mathbf{j}$ and $\mathbf{k}$ ), $500 \mu \mathrm{m}(\mathbf{e})$, and $1000 \mu \mathrm{m}$ (d)

the functional coupling of two specific cell types: PDECs and pancreatic islets.

Functional measurements in PDECs and pancreatic islets. PDECs) are reported to have the highest expression of CFTR in the body ${ }^{1,11-13}$. CFTR function in the pancreas has a critical role in maintaining fluid and $\mathrm{pH}$ within the pancreatic duct to deliver digestive enzymes secreted by acinar cells into the duodenum that are important for digestive function in the intestine. CFTR function was monitored in pancreatic ductal organoids in response to the cAMP-activating agonist forskolin (FSK; $10 \mu \mathrm{M}$ ). In this assay, CFTR function was reported as a measure of fluid secretion calculated by the ratio of luminal volume to that of the entire organoid ${ }^{26}$. During treatment with FSK, CFTR channels open, and chloride ions are pumped into the lumen creating an osmotic driving force for water to follow. Thus, fluid secretion is increased resulting in expansion of luminal volume. Fluid secretion was compared before and after treatment with FSK for $2 \mathrm{~h}$, as shown in Fig. 3a. Basal secretion of the ductal organoids was $60 \%$ before treatment and increased to $75 \%$ upon incubation with FSK. The graph was obtained using over 450 organoids derived from 21 pancreatitis patients. Alongside, we cultured ductal organoid-derived PDECs on a trans-well membrane and monitored CFTR function using short-circuit current $\left(I_{\mathrm{sc}}\right)$ measurement (Fig. 3b). The trans-electrical resistance was $1200 \Omega / \mathrm{cm}^{2}$ $\left(1.3 \times 10^{5}\right.$ of cells). By activating the CFTR channel with FSK, electrogenic movement of chloride ions from the apical side of these cells generated $I_{\mathrm{sc}}$ peak $\left(\Delta I_{\mathrm{sc}}=30 \mu \mathrm{A} / \mathrm{cm}^{2}\right)$ (Fig. 3b). The addition of CFTR inhibitor, CFTR inh-172 $(20 \mu \mathrm{M})$, caused dramatic decrease in the $I_{\text {sc }}$ (Fig. 3b). However, the context of CFTR function in the regulation of endocrine function by pancreatic islets remains to be elucidated. But before we investigated this possibility, we intended to monitor endocrine function in vitro. Pancreatic islets were isolated from the pancreatic remnant cell pellet of the same patient source as for PDECs and cultured in vitro using the methodology as described above (Fig. 3c). Pancreatic endocrine function consists of production of insulin ( $\beta$ cells) and glucagon ( $\alpha$ cells) from pancreatic islets to maintain an appropriate blood glucose level. The pancreatic islets were examined by immunofluorescence staining specific to insulin and glucagon (Fig. 3d). We observed arrangement of $\beta$ cells and $\alpha$ cells in the pancreatic islet marked by insulin (green) and glucagon (red), respectively (Fig. 3d). a Cells are located at the edge of the clustered pancreatic islets, while $\beta$ cells were distributed uniformly across the islet (Fig. 3d). We successfully monitored endocrine function in the pancreatic islets by measuring the concentration of insulin in the culture media in response to variable concentrations of glucose, $100 \mathrm{mg} / \mathrm{dL}$ (equivalent to normoglycemia) and $450 \mathrm{mg} / \mathrm{dL}$ (equivalent to hyperglycemia) (Fig. 3e). We observed that pancreatic islets secreted significantly more insulin when exposed to high glucose conditions; from $23.4 \mu \mathrm{LU} / \mathrm{mL}$ insulin in low glucose-containing media upon incubation for $1 \mathrm{~h}(3.3 \mu \mathrm{LU} / \mathrm{mL}$ at time $=0 \mathrm{~h})$ to $127 \mu \mathrm{LU} / \mathrm{mL}$ insulin in high-glucose-containing media during another $1 \mathrm{~h}$ incubation (Fig. 3e). In order to verify that endocrine function in islet cells obtained from pancreatitis patient is not impaired, we compared insulin secretion response to highly concentrated glucose $(450 \mathrm{mg} / \mathrm{dL})$ from non-pancreatic patient and pancreatitis patient (Supplementary Fig. 4). Increased insulin secretion upon exposure to high-glucose-containing media was observed in both the patients. Thus, the overall function in islet cells was not impaired in pancreatitis patient.

Therefore, we generated robust in vitro functional systems to monitor CFTR function from PDECs and endocrine function from pancreatic islets to set forth the stage to study CFRD.

A highly sensitive microfluidic device. Using human tissue has its limitations, including limited availability and a very low viable cellular yield. The short-circuit current $\left(I_{\mathrm{sc}}\right)$ assay is the goldstandard method to monitor CFTR function in real time; however, it requires approximately $1.3 \times 10^{5}$ cells, and takes approximately 2 weeks to achieve a fully covered-polarized monolayer of epithelial cells on the trans-well membrane $\left(33 \mathrm{~mm}^{2}\right)$. Here, we developed a highly sensitive microfluidic device to monitor CFTR function from PDECs and insulin secretion from pancreatic islets cultured on the chip as shown in Fig. 4 . The device, a single-channel chip (Fig. 4b), was designed to mimic pancreatic duct-like structure, which has branches with narrowing diameters (Supplementary Fig. 5). The chip was fabricated using standard photolithography and soft lithography, having dimensions $26.87 \mathrm{~mm}^{2}$ (area), $0.14 \mathrm{~mm}$ (thickness), and $3.76 \mathrm{~mm}^{3}$ (volume) for cell culture (Supplementary Fig. 6). We cultured PDECs (Fig. 4a) and pancreatic islets (Fig. 4c) to monitor CFTR function and insulin secretion, respectively. Total amount of cell culture media needed in the chip is only $56 \mu \mathrm{L}$, which includes $3.76 \mu \mathrm{L}$ for the cell culture chamber and $52 \mu \mathrm{L}$ for two side tubings. This is in contrast to the required $200 \mu \mathrm{L}$ (apical side) and $500 \mu \mathrm{L}$ (basolateral side) for an Ussing chamber. We have successfully monitored CFTR function in PDECs with $<10,000$ cells using the iodide efflux assay 3 days after seeding cells (Fig. 4d). In the first step of iodide efflux, cells were loaded with the iodide. Upon CFTR activation using FSK at time-point 10 min following a baseline, the iodide was pumped out of the cells through CFTR channel, which gave an iodide peak (60 \pm 18 $\mathrm{nM} / \mu \mathrm{L})$.

Using the single-channel chip, we were able to detect insulin secretion in pancreatic islets (Fig. 4e). Pancreatic islets were cultured on the chip with $100 \mathrm{mg} / \mathrm{dL}$ glucose-containing media (basal medium). We obtained $3 \mu \mathrm{LU} / \mathrm{mL}$ insulin from 15 pancreatic islets in the basal medium wash with no incubation. The concentration of secreted insulin increased from $27 \mu \mathrm{LU} / \mathrm{mL}$ $(1 \mathrm{~h}$ in the basal medium) to $106 \mu \mathrm{LU} / \mathrm{mL}(1 \mathrm{~h}$ in the highglucose-containing medium) (Fig. 4e). In this manner, we developed a highly sensitive microfluidic device to measure CFTR function in PDECs and insulin secretion in islets with small numbers of cells. 

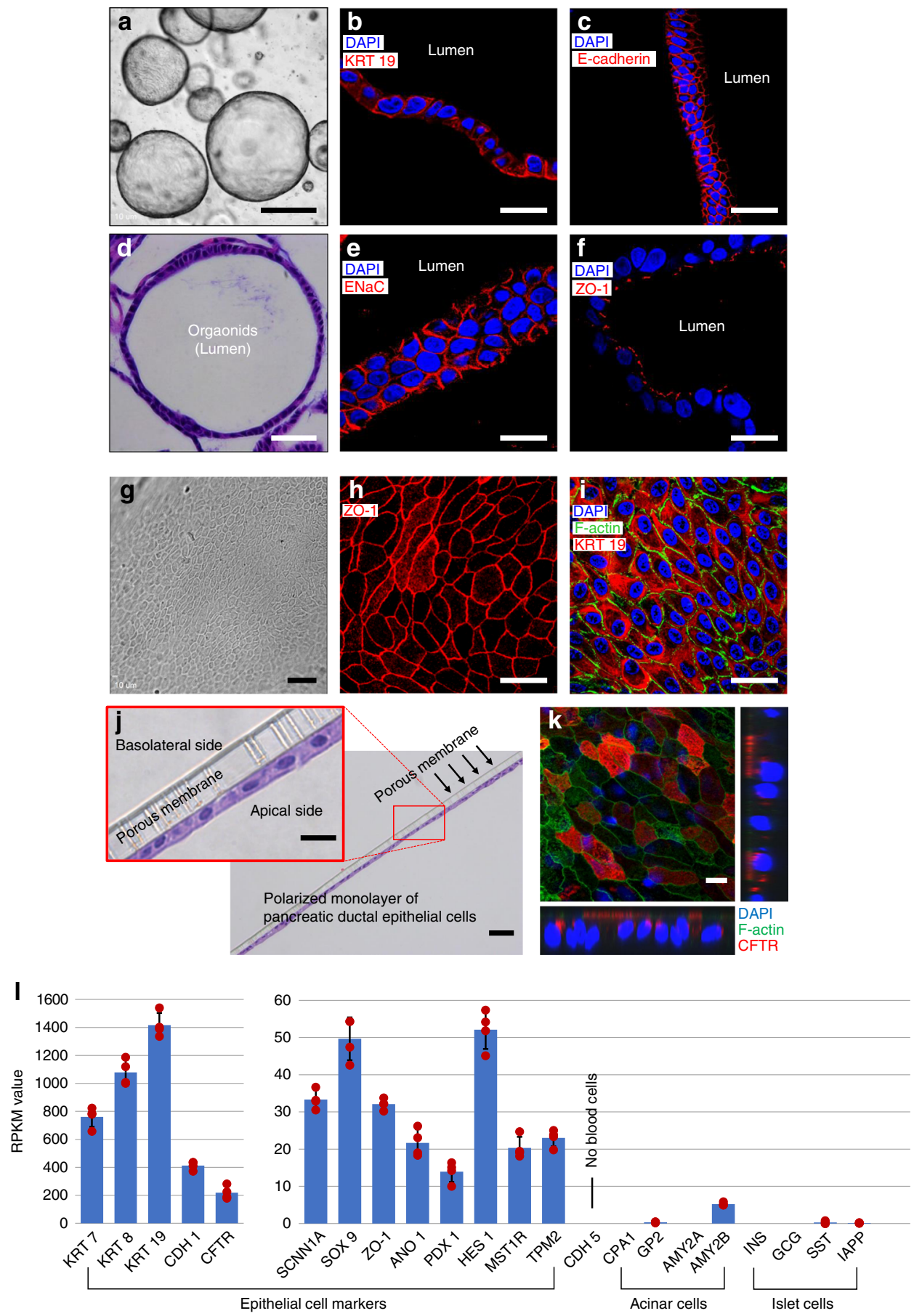

Fig. 2 Characterization of pancreatic ductal epithelial cells. (a-f, I: Characterization of organoids). a Characterization of pancreatic ductal organoids using epithelial cell markers, b cytokeratin 19 (KRT 19, c E-cadherin, e sodium transport channel (ENaC), and f ZO-1. d Hematoxylin and eosin (H\&E) image shows the orientation of pancreatic ductal epithelial cells in spheroid of the organoid. (g-k: Characterization of monolayer of pancreatic ductal epithelial cells (PDECs)). $\mathbf{g}$ Phase contrast and $\mathbf{j}$ H\&E images show monolayer of PDECs formed from the organoids. Monolayer of PDECs showed positivity for tight junction $\mathbf{h}$ ZO-1, i F-actin and KRT 19, and $\mathbf{k}$ cystic fibrosis transmembrane conductance regulator (CFTR). I RNA-sequencing data was obtained from the pancreatic ductal organoids and verified the PDEC origin $(n=4$ sample preparation from the same patient). Data are mean \pm SD. Scale bars: $10 \mu m(\mathbf{k})$, $20 \mu \mathrm{m}(\mathbf{b}, \mathbf{c}, \mathbf{e}, \mathbf{f}, \mathbf{h}-\mathbf{j}$ enlarge $), 50 \mu \mathrm{m}(\mathbf{d}, \mathbf{g}, \mathbf{j})$, and $500 \mu \mathrm{m}(\mathbf{a})$

Pancreas-on-a-chip to study CF-related disorders. We could detect that there is an interface between the ductal cells and islets based on $H \& E$ staining performed in a small piece $\left(1 \mathrm{~cm}^{2}\right)$ of non-treated tissue isolated from the head of the pancreas of a TPIAT patient (Fig. 5c) and immunostaining data in the same region, which was obtained from serial sections of the same sample, that showed CFTR-expressing ductal cells located in close proximity to insulin-expressing islets (Fig. 5a). Importantly, CFTR is only expressed in the PDECs, not in the pancreatic islets $^{12}$ (Supplementary Fig. 7). Given the cellular proximity between ductal cells and islets, we hypothesized that there is a functional coupling between these two cell types. To test this possibility, we developed a pancreas-on-a-chip involving coculturing of ductal epithelial cells and islets in two-cell culture 

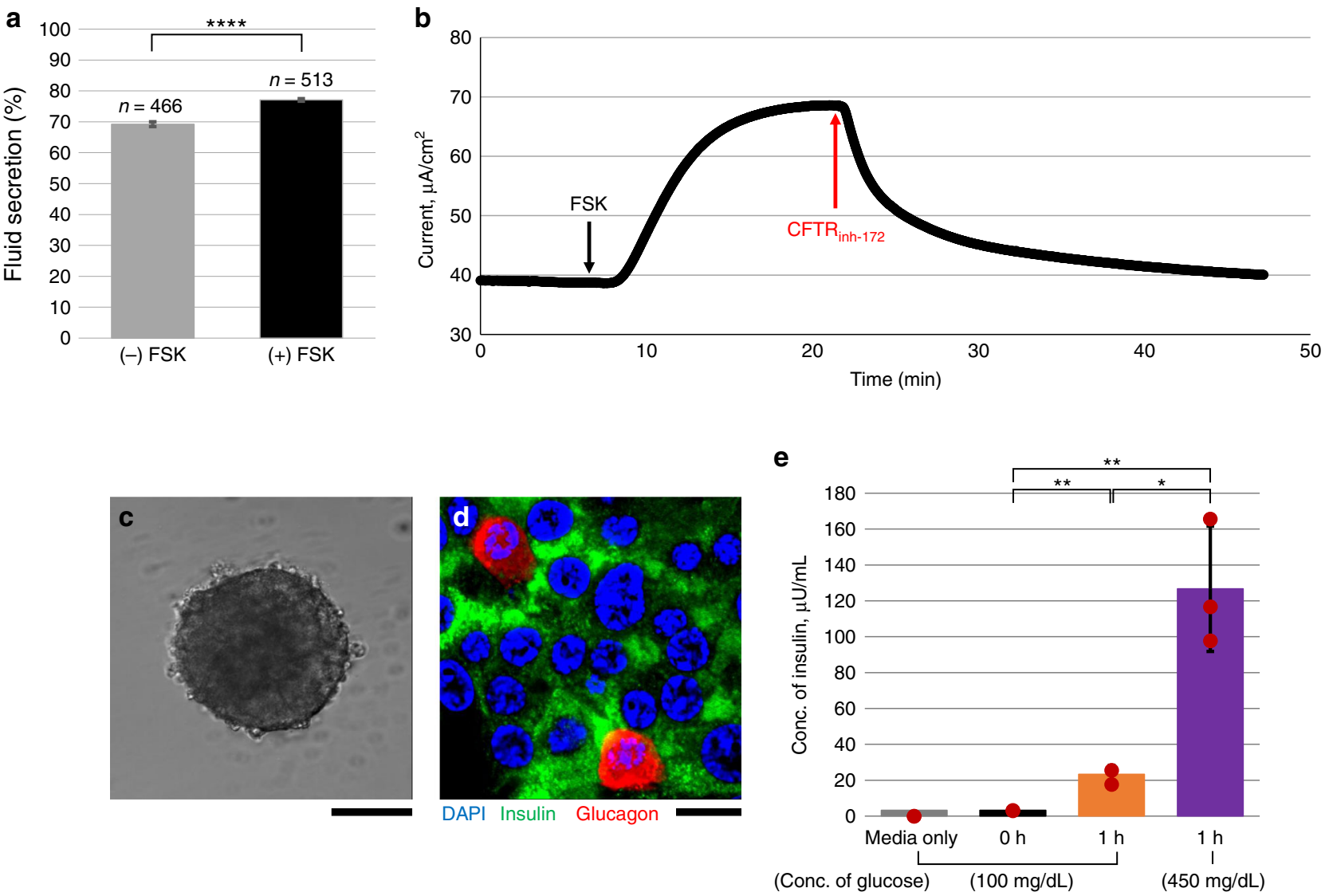

Fig. 3 Monitoring cystic fibrosis transmembrane conductance regulator (CFTR) channel function and endocrine function. CFTR channel function was monitored by stimulating CAMP with forskolin (FSK) using a fluid secretion assay for pancreatic ductal organoids ( $n>450$ organoids; from 21 pancreatitis patients; data are mean \pm SE) and $\mathbf{b}$ short-circuit current measurement in polarized monolayer of ductal epithelial cells grown on a trans-well filter. CFTR channel is activated by FSK and inhibited by CFTR $_{\text {inh-172. }}$ c Phase contrast image shows cultured pancreatic islet in vitro. $\mathbf{d}$ Pancreatic islets were examined by immunofluorescence detection of insulin (green) and glucagon (red). e Endocrine function was monitored by incubating pancreatic islets with different concentrations of glucose-containing media $(100$ and $450 \mathrm{mg} / \mathrm{dL})$ for $1 \mathrm{~h}$ serially. Pancreatic islets were stimulated by high glucose $(n=3$ sample preparation from the same patient; data are mean \pm SD). Scale bars: $100 \mu \mathrm{m}$ (c) and $20 \mu \mathrm{m}(\mathbf{d})$. ( $p$ values from one-way analysis of variance (ANOVA) and adjust using Bonferroni factor: ${ }^{\star}<0.01,{ }^{\star \star}<0.005,{ }^{\star \star \star \star}<1.0 \times 10^{-20}$ )

chambers separated by a thin layer of porous membrane $(<10 \mu \mathrm{m}$ thickness) (Fig. 5b and Supplementary Fig. 8). The PDECs were cultured on the top chamber and pancreatic islets were seeded in the bottom chamber (Fig. 5d). Next, we tested how CFTR function may affect insulin secretion from the islet cells in this doublechannel chip system. We measured secreted insulin in 1-h increments from pancreatic islets in the bottom chamber following stimulation or inhibition of CFTR channel function (Fig. 5e). Stimulation of CFTR channels in PDECs in the top chamber did not show significant changes. On the other hand, upon inhibition of CFTR channel function in PDECs using CFTR $_{\text {inh-172 }}$ (Chip B; $20 \mu \mathrm{M}, 1 \mathrm{~h}$ ), insulin secretion was significantly decreased $(53.7 \% ; \Delta 191.4 \mu \mathrm{LU} / \mathrm{mL})$. We examined if the CFTR inhibitor directly affects insulin secretion from the islet cells (Supplementary Fig. 9). We cultured islet cells in the double-channel chip without PDECs (Supplementary Fig. 9a) or co-cultured with PDECs lacking pores on the membrane to perturb communication between PDECs and islet cells (Supplementary Fig. 9b) and added $20 \mu \mathrm{M}$ CFTR $_{\text {inh-172 }}$ to the top chamber. We observed that inhibition of CFTR under these conditions did not influence insulin secretion. Insulin secretion from the islet cells was not altered in the presence of FSK $(10 \mu \mathrm{M})$ and CFTR $_{\text {inh-172 }}(20 \mu \mathrm{M})$ (Supplementary Fig. 10). FSK stimulation did not significantly alter insulin secretion from the islet cells maintained in the basal medium $(100 \mathrm{mg} / \mathrm{dL}$ glucose $)$. At the end of the experiment, islet cells were directly exposed to highglucose-containing media $(450 \mathrm{mg} / \mathrm{dL}$ glucose; Chip A and Chip B) to verify its responsiveness to the glucose challenge, suggesting that the endocrine function was not impaired. Insulin secretion increased in both chips (Chip A: $\Delta 60.7 \mu \mathrm{LU} / \mathrm{mL}$ and Chip B: $\Delta 181.4 \mu \mathrm{LU} / \mathrm{mL}$ ) and the amount of insulin secreted was higher than before stimulation or inhibition of CFTR function in PDECs.

To further consolidate the possibility that CFTR function directly affects insulin secretion, we examined the cell-cell functional correlation between PDECs and islet cells derived from pancreatitis/CF patient, who was diagnosed with very mild CF and underwent TPIAT (Supplementary Table 1). The patient has $\Delta F 508$ (allele 1), R117H (allele 2), and heterozygote for SPINK1 mutation. This patient was diagnosed to have mild CF and has some CFTR function as demonstrated by the mild phenotype (i.e., body mass index: 19.84; sweat chloride: $51 \mathrm{mmol} /$ $\mathrm{L}$; forced expiratory volume in $1 \mathrm{~s}$ predicted: $114 \%$ and is not diabetic). Additionally, we monitored CFTR function using fluid secretion assay and endocrine function using enzyme-linked immunosorbent assay (ELISA) as described earlier prior to coculture of the two cell types in pancreas-on-a-chip. We observed that the pancreatic ductal organoids showed partially impaired CFTR function (20\% lower than non-CF pancreatitis patient in basal secretion and under 5.3\% in FSK-stimulated secretion). Islet 


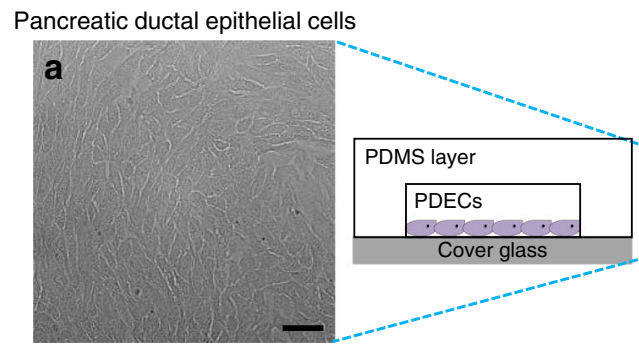

d

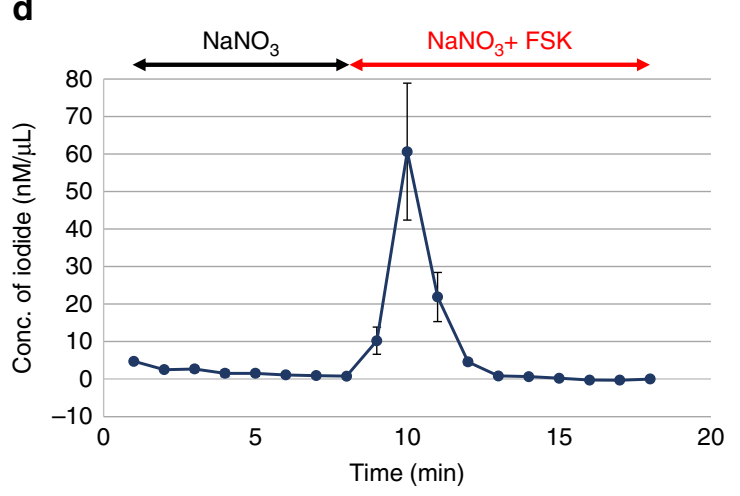

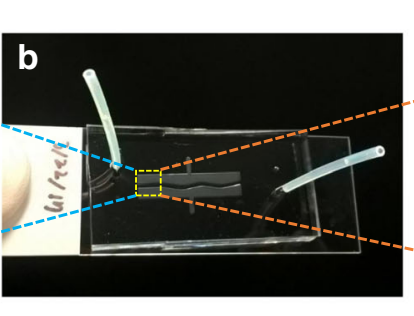
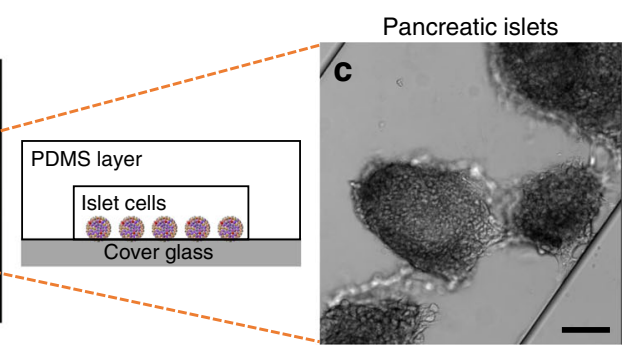

e

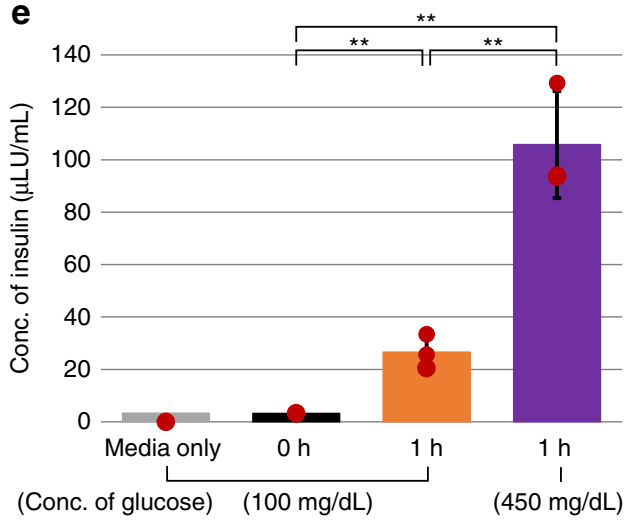

Fig. 4 A unique microfluidic device. b A microfluidic device, single-channel chip, was designed to mimic pancreatic duct-like structure with branches and narrowing diameters. a) Pancreatic ductal epithelial cells (PDECs) were cultured in the chip and (d) cystic fibrosis transmembrane conductance regulator (CFTR) function was monitored using iodide efflux assay with $<10,000$ PDECs $(n=3)$. e Endocrine function was monitored with 15 pancreatic islets ( $n=$ 3) c by incubating with 100 and $450 \mathrm{mg} / \mathrm{dL}$ glucose-containing media for $1 \mathrm{~h}$ serially. Secreted amount of insulin was measured using enzyme-linked

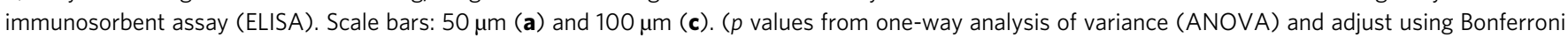
factor: ${ }^{\star \star}<0.005 ; n=3$ number of chips; data are mean \pm SD)

cells secreted insulin in response to the glucose challenge (Supplementary Fig. 10a, b). We co-cultured PDECs and islet cells in pancreas-on-a-chip and measured insulin secretion from the islet cells as described earlier. We observed similar trend that inhibition of CFTR function affected endocrine function. Insulin secretion was decreased in pancreatitis/CF patient-derived pancreas-on-a-chip by $7.9 \%$, but it was not significant (Supplementary Fig. 10c, d).

Overall, using this unique pancreas-on-a-chip device, we demonstrated that ductal cells and islets are functionally coupled, a first-of-a-kind observation that CFTR plays a role in directly regulating insulin secretion. This observation is directly relevant to CFRD in which there is a loss of CFTR function.

\section{Discussion}

We have successfully isolated patient-derived pancreatic ductal organoids following TPIAT, and we have generated a freezing and reviving protocol for pancreatic ductal epithelial cells. Pancreatic ductal organoids demonstrated growth into large spheres over time. The organoids cultured in $3 \mathrm{D}$ matrix positions us to efficiently harvest pure pancreatic ductal epithelial cells among multiple cell types that are present in the pancreatic remnant cell pellet. The organoids were grown effectively from a limited number of cells to form a functional unit. The 3D organoid formation with luminal area internally has been observed in other organs, including lung ${ }^{27}$, liver ${ }^{28}$, and intestine ${ }^{29}$. However, this finding is our repeated observation of duct-like formation from the pancreatic ductal organoids. While the mechanism is currently unclear, further investigation of this ductal formation may elucidate mechanisms involved in the development of the pancreatic duct in vivo.

Pancreas-on-a-chip mimics in situ pancreatic cell function and interface compared to conventional human cell culture model.
The chip allows to mimic fluid flow in vivo by setting a perfusion system in a cell culture incubator or on a microscope, relevant mechanical cues in cellular signaling, and allows tissue-issue interface (i.e., duct-islet) to study cell-cell signaling ${ }^{30}$. Pancreason-a-chip helps answer the fundamental question in CFRD: is loss of CFTR function in PDECs primary to CFRD development. Based on our data, it is indeed the case. Surprisingly, the absolute amount of insulin was around $50 \%$ decreased during inhibition of CFTR channel function. In the human pancreas, the organ system is extremely complex in physiological and pathological perspectives. However, this finding tells us that CFTR channel function plays a critical role in maintaining endocrine function and may provide critical insight into the etiology of CFRD. To investigate the cross-talk between PDECs and pancreatic islets, metabolism studies of these two cell types may need to be performed.

CFRD is a serious complication in CF patients who in general have disordered glucose metabolism with increasing risk with advancing age. Using this in vitro chip model, we can study CFRD and glucose imbalance in CF individuals, assay variability in the glucose measures in these individuals, determine correlation of glucose levels with the CFTR mutation type, and test small-molecule interventions (i.e., approved CFTR modulators) that may improve glucose abnormalities in the patient samples. Our data based on the effect of CFTR-specific inhibitor and lack of function mutation in CFTR strongly suggests that CFTR function modulates insulin secretion that underlies the pathology of CFRD.

This patient-derived in vitro model system also allows the development of personalized medicine with highly sensitive measurements of epithelial and/or endocrine functions from the pancreatic cells. Because the cells cultured in the chip are all patient derived, we can easily and quickly obtain other clinically relevant measures using this model in safe manner. Alcohol abuse 

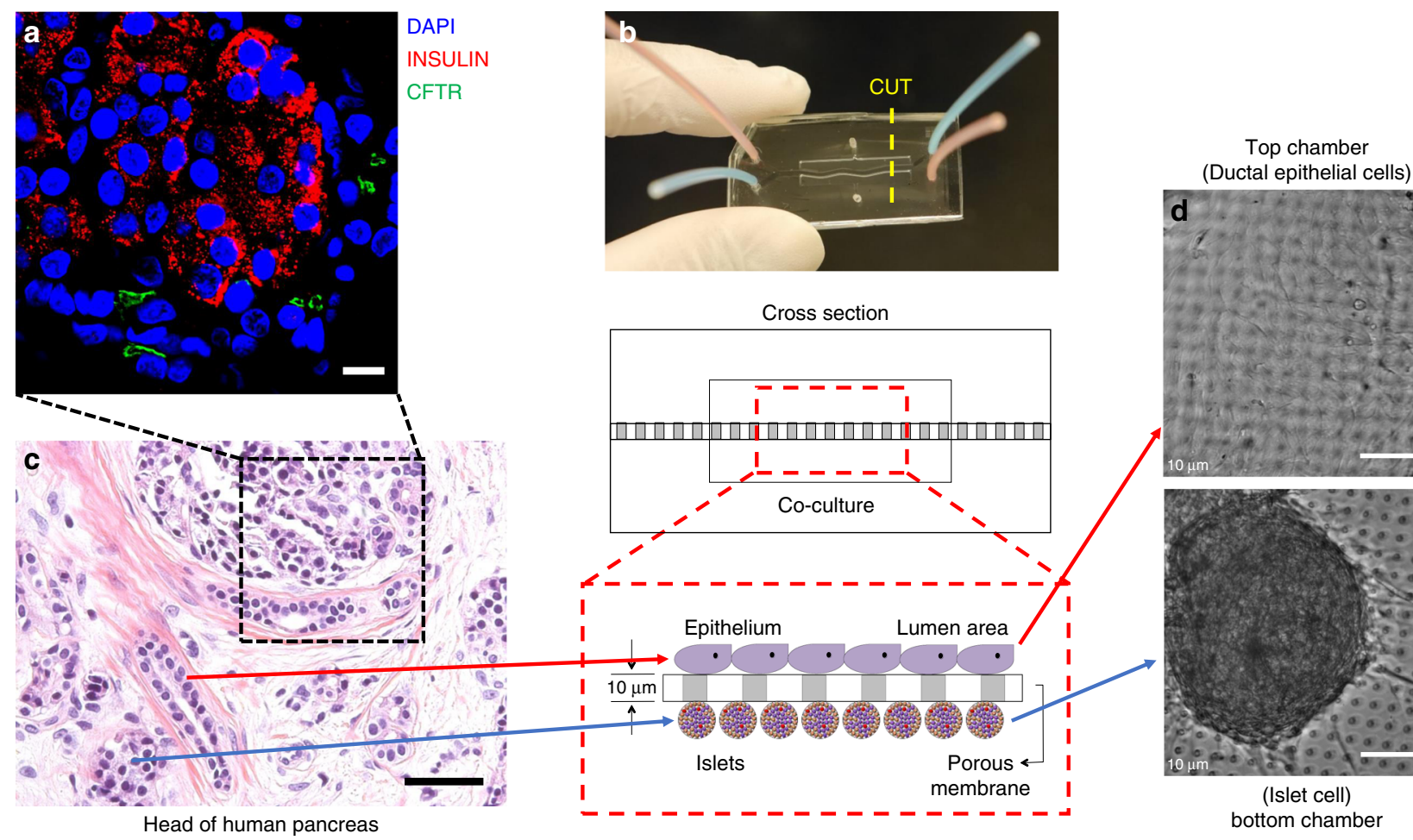

(Ductal epithelial cells)

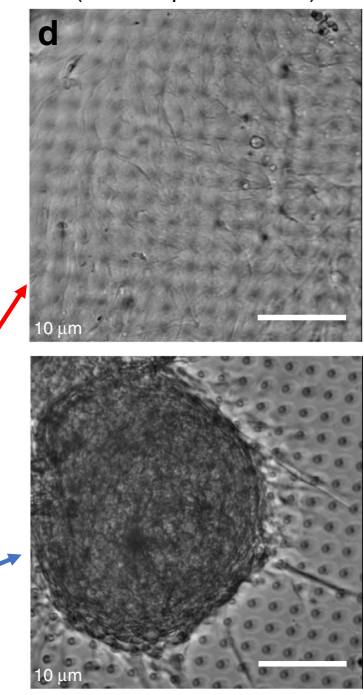

(Islet cell)

bottom chamber

e

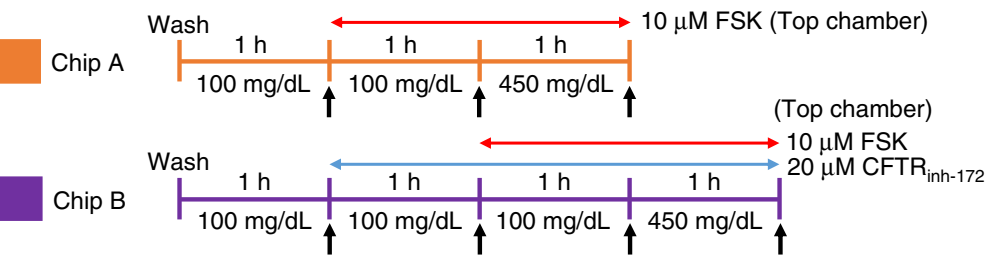

$(\uparrow$ : Collect supernatant from bottom chamber)

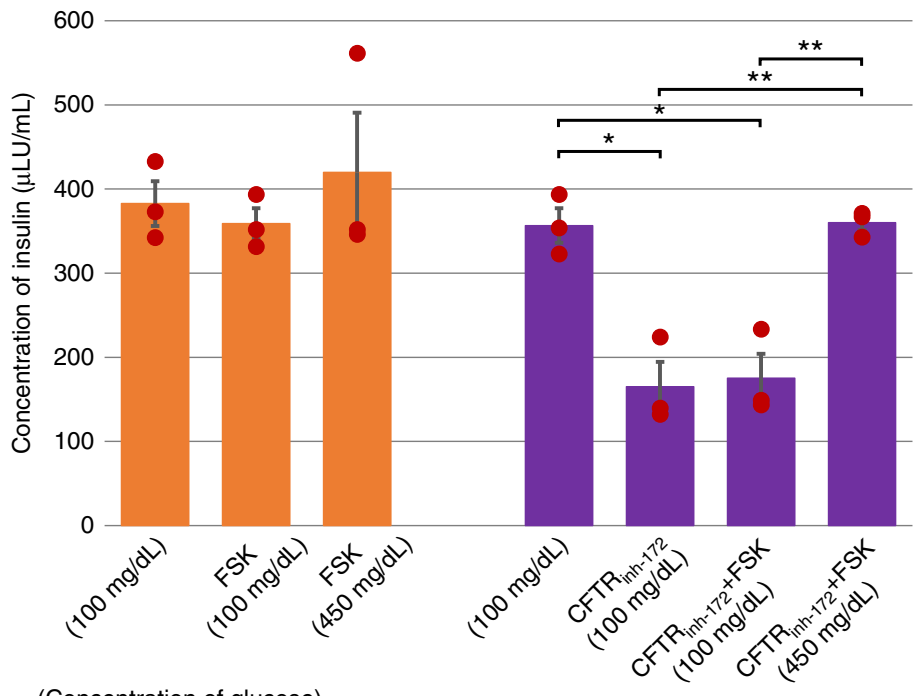

(Concentration of glucose)

Fig. 5 Pancreas-on-a-chip to study cystic fibrosis-related diabetes (CFRD). A small piece of non-treated head of pancreas was obtained and examined by a immunofluorescence microscopy with insulin and cystic fibrosis transmembrane conductance regulator (CFTR) and $\mathbf{c}$ hematoxylin and eosin (H\&E) stain. It shows that pancreatic islets are located in close proximity to the pancreatic duct. To mimic pancreatic structure and function, $\mathbf{b}$ pancreas-on-a-chip has been developed that is comprised of two-cell culture chambers and a thin layer of porous membrane. $\mathbf{d}$ Pancreas-on-a-chip allows us to co-culture pancreatic ductal epithelial cells (PDECs) on the top chamber with pancreatic islets in the bottom chamber. e Endocrine function of islet cells was monitored with stimulation or inhibition of CFTR function in PDECs on the top chamber. We observed that CFTR channel function has a direct effect on the endocrine function. Secreted insulin was dramatically decreased (53.7\%) by inhibition of CFTR function of PDECs. This in vitro model system, pancreas-ona-chip, allows us to study cell-cell interaction. Scale bars: $10 \mu \mathrm{m}(\mathbf{a}), 50 \mu \mathrm{m}$ (c), and $100 \mu \mathrm{m}$ (d). ( $p$ values from one-way analysis of variance (ANOVA) and adjust using Bonferroni factor: ${ }^{\star}<0.05,{ }^{\star \star}<0.005$; number of chips: Chip A $(n=3)$ and Chip B $(n=4)$; data are mean \pm SE) 
has been reported to lead to dysfunction and degradation of CFTR protein on the apical membrane of the epithelium ${ }^{31}$. Using this chip model, we can monitor CFTR function and/or endocrine function in response to alcohol in a dose-dependent manner that is not possible in patients.

The microfluidic device can be set up for multiple analyses, including functional assays and microscopic measurements in real time. This in vitro model system will facilitate drug discoveries. However, the polydimethylsiloxane (PDMS) used for the cell culture chambers has a challenging property, which is that the hydrophobic PDMS absorbs hydrophobic small molecules ${ }^{32,33}$. After oxygen plasma treatment, it changes to highly hydrophilic $^{34}$. However, it recovers to hydrophobic over time ${ }^{35}$. The hydrophobic surface interferes with cell adhesion on the substrate $^{36}$. Alternatively, other materials for fabricating the microfluidic device have been adopted, such as poly methyl methacrylate ${ }^{37}$, acrylonitrile butadiene styrene copolymer $^{33}$, cyclic olefin copolymer ${ }^{38}$, and styrene ethylene butylene styrene $^{39}$. However, those materials also have limitations when mimicking human organ systems due to their rigidity and brittleness, leading to a difficult fabrication process. The Kang laboratory has demonstrated that PDMS-based microfluidic devices can be maintained as a hydrophilic surface for weeks after treatment with oxygen plasma ${ }^{34}$. Other groups showed that solgel-modified PDMS 40 and bovine serum albumin-coated -PDMS ${ }^{41}$ can minimize absorbance of hydrophobic drugs by PDMS. Alternatively, collagen coating of the chamber can be utilized to increase cell adhesion, as we used in our model system. Although these limitations are yet to be overcome, PDMS is still the best material to mimic organ systems.

In summary, we have isolated and cultured patient-derived pancreatic cells, PDECs, and pancreatic islets from the same patient. This efficient and highly reproducible method allows us to widely study relevant pancreatic disorders. Moreover, the in vitro model system, pancreas-on-a-chip, helps us to investigate the cross-talk between PDECs and islet cells in the development of disease pathologically and physiologically. This pancreas-on-achip model system, with its highly sensitive profile, can allow for early diagnosis and individual diagnosis that may help prevent or reduce the progression of disorders such as CFRD, and additionally, can afford the opportunity for drug discovery and personalized medicine in such disorders.

\section{Methods}

Human studies. Human tissue, pancreatic remnant cell pellets were collected according to standard research protocols approved by the Institutional Review Board and Department of Pathology at Cincinnati Children's Hospital (IRB: 20146279; renewed 27/11/2017).

Cell culture media. For PDECs, advanced Dulbecco's modified Eagle's medium/ nutrient mixture F12 (DMEM/F12) (Invitrogen; \#12634010) with $10 \mathrm{mM}$ HEPES (Invitrogen; \#15630-080), GlutaMAX (1×; Invitrogen; \#35050-061), and penicillin streptomycin (PS) (1×; Invitrogen; \#15140-122) was used as base organoid media (I). Organoid media (II) contains N2 (1×; Invitrogen; \#17502-048), B27 (1×; Invitrogen; \#17504-044), and $1 \mathrm{mM} \mathrm{N}$-acetylcysteine (Sigma; \#A7250-100G) in organoid media (I). Organoid media (III) contains growth factors, supplements $100 \mathrm{ng} / \mathrm{mL}$ epidermal growth factor (R\&D System; \#236-EG-200), $50 \mathrm{ng} / \mathrm{mL} \mathrm{R}$ spondin (R\&D System; \#4645-RS-025/CF), and $100 \mathrm{ng} / \mathrm{mL}$ Noggin (R\&D System; \#6057-NG-025) in organoid media (II). ROCK inhibitor, $10 \mu \mathrm{M}$ Y-27632 (BD Biosciences; \#562822), was added for the first 4 days followed by isolation of pancreatic ductal epithelial cells.

Islet cells were cultured in RPMI-1640 (Invitrogen; \#61870036) containing PS (1×), 10\% fetal bovine serum (FBS) (Atlanta Biologicals; \#S11150 premium), and $10 \mu \mathrm{M}$ Y-27632 for the first day of isolation. The RPMI-1640 media were switched to low glucose-containing DMEM (Invitrogen; \#11885-084; $100 \mathrm{mg} / \mathrm{dL}$ glucose) with PS $(1 \times)$ and $10 \%$ FBS from the second day of isolation. High-glucosecontaining DMEM (Invitrogen; \#11960-044; $450 \mathrm{mg} / \mathrm{dL}$ glucose) was used to stimulate pancreatic islets.
Isolation of pancreatic ductal organoids and islet. Pediatric patients with severe acute recurrent or chronic pancreatitis undergo TPIAT. During the TPIAT, the excised pancreas was surgically dissected and digested to isolate pancreatic islets for infusion into the liver through the portal vein. We obtained discarded pancreatic remnant cell pellets following isolation of pancreatic islets. The pancreatic remnant cell pellet still contains pancreatic islets, ductal epithelial cells, and acinar cells (Fig. 1c). From the remnant cell pellet, we isolated pancreatic ductal tissues by microscopic dissection under a stereo microscope (Leica; \#M165FC) (Fig. 1e). The pancreatic remnant cell pellet was prepared on a $60-\mathrm{mm}$ dish containing phosphate-buffered saline (PBS) by dissecting a white cluster (Fig. 1b; circle, Fig. 1d) using two forceps until the appearance of pancreatic duct-like structure remained. Then, holding the cluster using one forcep the pancreatic duct is gently pulled out using the other forceps. The pancreatic duct is extracted smoothly, because surrounding connecting tissues were uniformly oriented along the length of the duct. Microdissection scissors were used to remove other cell types attached to the ductal tissues, if necessary. Isolated ductal tissues were treated with $2 \mathrm{mM}$ EDTA (Invitrogen; \#15575) in $10 \mathrm{~mL}$ PBS on a shaker (Nutator; \#421105) at $4{ }^{\circ} \mathrm{C}$ for $40 \mathrm{~min}$. The tissues were filtered through a $70 \mu \mathrm{m}$ strainer (Falcon; \#352350) to remove the EDTA solution, and all tissues were transferred to fresh $15 \mathrm{~mL}$ tube containing $10 \mathrm{~mL}$ PBS. The tube was shaken mechanically to help separate ductal epithelial cells apart from the tissues. The supernatant was then filtered through a fresh $70 \mu \mathrm{m}$ strainer and $10 \%$ FBS was added to stop the proteolytic activity. Supernatant was discarded after centrifugation (Beckman Coulter; \#Allegra X-14R) at $233 \times g$ for $3 \mathrm{~min}$. Organoid media (II) were added and the ductal epithelial cells were re-suspended by pipetting gently. Matrigel (Corning; \#356231) was added at a ratio of $2: 3 \mathrm{vol} \%$ growth media to Matrigel and mixed well avoiding any bubbles. Matrigel $(50 \mu \mathrm{L})$ was plated with ductal cells on a substrate and incubated at $37^{\circ} \mathrm{C}$ for $15 \mathrm{~min}$ for solidification of the Matrigel. The Matrigel was covered with $500 \mu \mathrm{L}$ organoid media (III) containing growth factors. For the first 4 days, $10 \mu \mathrm{M}$ Y-27632 of ROCK inhibitor was added to help cellular recovery from cellular aggregates ${ }^{42,43}$. Organoid media (III) were refreshed every other day.

Pancreatic remnant cell pellets contain leftover islet cells after isolation of islets for infusion. Islet cells can be easily identified by dithizone staining $(100 \mu \mathrm{g} / \mathrm{mL})$, because dithizone binds to the zinc ion presented in insulin secreted by $\beta$ cells in the pancreatic islets ${ }^{25}$. Dithizone solution is green, but it turns to red when it binds to the zinc ion (Fig. 1d, g). Islet cells were transferred gently to a fresh plate using a $200 \mu \mathrm{L}$ pipette and cultured in RPMI-1640 media containing $10 \mu \mathrm{M}$ Y-27632 for the first day. Next day, the media were switched to low glucose-containing DMEM $(100 \mathrm{mg} / \mathrm{dL})$ and refreshed every other day.

Obtaining monolayer of pancreatic ductal epithelial cells. Pancreatic ductal organoids were grown over time in Matrigel (Fig. 1h). The mechanism is unknown, but we continuously observed that when the ductal organoids contact the surface, they start to form duct-like structures and then a complete monolayer (Supplementary Fig. 2). To assist forming a monolayer of PDECs, the Matrigel was broken down by pipetting with $1 \mathrm{~mL}$ organoid media (I) when the average diameter of the organoids reached $500 \mu \mathrm{m}$. Organoids were then transferred to $1.5 \mathrm{~mL}$ tube by using a $200 \mu \mathrm{L}$ pipette. After centrifugation at $8600 \times g$ for $3 \mathrm{~min}$ (Eppendorf microcentrifuge; \#5418), the supernatant was discarded and the organoid media (III) containing $10 \mu \mathrm{M}$ Y-27632 were added to the pellet. Organoids were then plated on a fresh dish or trans-well membrane. Care must be taken to separate the Matrigel completely from the organoids, or they will not adhere, and will not survive.

Freezing and reviving pancreatic ductal epithelial cells. To cryopreserve PDECs, PDEC monolayers were trypsinized with $0.5 \%$ trypsin EDTA (1×; Invitrogen; \#15400-054) at $37^{\circ} \mathrm{C}$ for 10 min to detach cells after washing cells with PBS and transferred to $15 \mathrm{~mL}$ tube containing $5 \mathrm{~mL}$ organoid media (I) with $10 \%$ FBS and $10 \mu \mathrm{M}$ Y-27632. Cell pellets were obtained after centrifuging at $233 \times g$ for $5 \mathrm{~min}$. The supernatant was discarded and cells were re-suspended with freezing media (Invitrogen; \#12648010) containing $10 \mu \mathrm{M}$ Y-27632. Epithelial cells were transferred to a cryopreservation tube and placed on dry ice immediately and stored at $-80^{\circ} \mathrm{C}$. For long-term storage, the cells were stored in liquid nitrogen.

To revive PDECs, the cells were thawed quickly at $37^{\circ} \mathrm{C}$ and all supernatants were transferred to $15 \mathrm{~mL}$ tube containing $5 \mathrm{~mL}$ organoid media (I) with $10 \mu \mathrm{M}$ Y 27632. After centrifugation at $233 \times g$ for $5 \mathrm{~min}$, the supernatant was discarded. Appropriate organoid media (II) with Matrigel were added and $50 \mu \mathrm{L}$ Matrigel was plated with cells on plates to form organoid structure as before. The Matrigel was covered with organoid media (III) containing $10 \mu \mathrm{M}$ Y-27632 after incubation at $37^{\circ} \mathrm{C}$ for $15 \mathrm{~min}$

Fabrication of pancreas-on-a-chip. Our customized microfluidic device was designed to mimic ductal structure having branches with narrowing diameters (Supplementary Fig. 5). The design was drawn using the AutoCAD software. The chip was fabricated through the standard photolithography and soft lithography techniques (Supplementary Fig. 6). Initially, the silicon wafer was washed with acetone, isopropanol (IPA) and water. It was placed on a hot plate at $60{ }^{\circ} \mathrm{C}$ for $10 \mathrm{~min}$ to dry thoroughly after air drying. After cooling down to room temperature, a negative photoresist SU-8 (Microchem; \#Y131269) was applied to the wafer using 
a spin coater (Specialty Coating Systems; \#6800) by the following process: (1) Place the wafer on the vacuum chuck of the spin coater and drop appropriate SU-8 on the wafer. (2) Ramp up to $500 \mathrm{rpm}$ for $10 \mathrm{~s}$ and hold for $10 \mathrm{~s}$. (3) Increase the speed to $1000 \mathrm{rpm}$ for $10 \mathrm{~s}$ and hold it for $15 \mathrm{~s}$ for $140 \mu \mathrm{m}$ thickness of cell culturing chamber in the chip. (4) Speed down to $0 \mathrm{rpm}$ for $10 \mathrm{~s}$. The wafer was placed on the hot plate and baked at $65^{\circ} \mathrm{C}$ for $10 \mathrm{~min}$ and at $95^{\circ} \mathrm{C}$ for $30 \mathrm{~min}$ serially. The wafer was exposed to ultraviolet (UV) light (wavelength: $365 \mathrm{~nm}$; exposure energy: $240 \mathrm{~mJ} / \mathrm{cm}^{2}$ ) through a patterned photomask for $20 \mathrm{~s}$ after cooling down to room temperature. The wafer was baked on the hot plate at $65^{\circ} \mathrm{C}$ for $1 \mathrm{~min}$ and at $95^{\circ} \mathrm{C}$ for $20 \mathrm{~min}$ and was cooled down to room temperature. The wafer was immersed into SU-8 developer (Fisher Scientific; \#NC9901158) for development process of unexposed area to UV light. After completion of development, the wafer was washed with IPA and dried with filtered air. The patterned silicon wafer was then baked on the hot plate at $150{ }^{\circ} \mathrm{C}$ for $30 \mathrm{~min}$. After cooling down to room temperature, the patterned wafer can be used as a mold. These standard photolithography procedures were carried out in in a 100-class clean room.

For this microfluidic device, we used flexible, transparent, and low-cost materials, PDMS (Ells Worth Adhesive; \#4019862). We mixed viscous PDMS with curing kit at the ratio of 10:1 (wt\%) and degassed in a desiccator to remove bubbles. In the meantime, the patterned silicon wafer was treated with trichloro silane (Sigma-Aldrich; \#448931) for $30 \mathrm{~min}$ in another desiccator to assist peeling off the patterned PDMS layer from the wafer. The uncured PDMS was cast onto the wafer and cured at $60^{\circ} \mathrm{C}$ for at least $4 \mathrm{~h}$. The solidified patterned PDMS layer was peeled off from the wafer and holes were created at both ends of the cell culture area for seeding and feeding cells. The patterned PDMS layer and a cover glass were treated with oxygen plasma for $30 \mathrm{~s}$ using Tergeo Plasma Cleaner (PIE Scientific) and immediately assembled together. It was placed on the hot plate at $120^{\circ} \mathrm{C}$ for $30 \mathrm{~min}$ to seal completely that is a single-channel chip. The activated surface of the patterned PDMS layer and cover glass by plasma treatment becomes highly hydrophilic with polar characteristics ${ }^{34}$. This enhances the bonding process of the two surfaces.

Pancreas-on-a-chip is comprised of top and bottom layers for cell culture chambers and a thin layer of porous membrane to separate the two chambers as double-channel chip. Patterned PDMS layers of top and bottom chambers are prepared as described previously for single-channel chip. Holes were created through the PDMS layer of the top chamber for seeding and feeding cells before assembly with the porous membrane. For the thin layer of porous membrane, a mold was fabricated of uniformly arranged cylinders, with $10 \mu \mathrm{m}$ diameters, $25-\mu \mathrm{m}$ gaps, and $40-\mu \mathrm{m}$ thickness, on a silicon wafer through the photolithography. The wafer is coated with trichloro silane in the desiccator for $30 \mathrm{~min}$. In the meantime, RTV615 (Momentive; \#9480), which shows large linear behavior of stain and promotes fabrication of a thin layer uniformly comparing to $\mathrm{PDMS}^{44,45}$, is mixed with a curing kit at the ratio of 5:1 (wt\%) and degassed in the desiccator for $30 \mathrm{~min}$. The patterned wafer was placed on the spin coater and spun after covering the pattern with degassed RTV615 as the standard for $10 \mu \mathrm{m}$ thickness of porous membrane; thus, (1) ramp up to $500 \mathrm{rpm}$ for $10 \mathrm{~s}$ and hold for $10 \mathrm{~s}$; (2) increase the speed to $3000 \mathrm{rpm}$ for $10 \mathrm{~s}$ and hold it for $5 \mathrm{~min}$; (3) speed down to $0 \mathrm{rpm}$ for $10 \mathrm{~s}$. Leave the wafer at room temperature for $10 \mathrm{~min}$ for uniform surface and incubate at $60^{\circ} \mathrm{C}$ for $10 \mathrm{~min}$ for partial solidification of the surface. After incubation, the top PDMS layer was placed, patterned face down, directly onto the cylinders and slightly pressed onto the PDMS layer for contacting the surface of top layer to the partially cured RTV615. The top chamber is incubated with the porous membrane on the wafer overnight and cooled down to room temperature. The top chamber with the porous membrane is peeled from the wafer and holes created through the porous membrane to connect to the bottom chamber only. The top chamber and bottom chamber are aligned after oxygen plasma treatment and placed on the hot plate at $120^{\circ} \mathrm{C}$ for $30 \mathrm{~min}$ to seal the double-channel chip.

Before seeding cells, the cell culture chambers were sterilized with $70 \% \mathrm{EtOH}$ for $10 \mathrm{~min}$ and washed with autoclaved water using a needle (BD Biosciences; \#305175; $20 \mathrm{G}$ ) and syringe (BD Biosciences; \#309657; $3 \mathrm{~mL}$ ). The chambers were coated with $50 \mu \mathrm{g} / \mathrm{mL}$ collagen (Sigma-Aldrich; \#C3867-1VL) for $1 \mathrm{~h}$ at $37^{\circ} \mathrm{C}$ and washed with PBS to increase cell adhesion. The microfluidic device was connected to a peristaltic pump (Cole-Parmer; \#ISMATEC Reglo ICC) with tubing (ColeParmer; \#97619-09) and supplied organoid growth media (III) at the flow rate of $1 \mu \mathrm{L} / \mathrm{min}$ to feed cells continuously. To feed cells manually, a syringe and needle was used.

Culture cells in the microfluidic device. Monolayers of PDECs were treated with $0.5 \%$ Trypsin EDTA $(1 \times)$ at $37^{\circ} \mathrm{C}$ for $10 \mathrm{~min}$ and floating cells were transferred to a $15 \mathrm{~mL}$ tube containing $5 \mathrm{~mL}$ organoid media (I) with $10 \%$ FBS and $10 \mu \mathrm{M}$ $\mathrm{Y}-27632$. The supernatant was discarded after spinning down at $233 \times g$ for $5 \mathrm{~min}$ $\left(4{ }^{\circ} \mathrm{C}\right)$ and cells were re-suspended with $120 \mu \mathrm{L}$ organoid media (III) containing $10 \mu \mathrm{M}$ Y-27632. Cells were transferred $\left(2 \times 10^{5}\right.$ cells $/ \mathrm{mL} ; 10,000$ cells/chip $)$ in the cell culture chamber coated with collagen $(50 \mu \mathrm{g} / \mathrm{mL})$ using a syringe and needle through a tubing $(5 \mathrm{~cm}$ length) inserted through the PDMS layer. After overnight incubation at $37^{\circ} \mathrm{C}, 5 \% \mathrm{CO}_{2}$ media were refreshed.

Pancreatic islets in 24-well plate were washed with PBS and incubated with $200 \mu \mathrm{L} 0.5 \%$ Trypsin EDTA $(1 \times)$ at $37^{\circ} \mathrm{C}$ for $3 \mathrm{~min}$ for trypsinization. Pancreatic islets were transferred to a $1.5 \mathrm{~mL}$ tube filled with culture media. The supernatant was discarded after centrifugation at $8600 \times g$ (microcentrifuge) for $3 \mathrm{~min}$ and cells were re-suspended with $120 \mu \mathrm{L}$ media. Pancreatic islets were transferred (300 islets/ $\mathrm{mL} ; 15$ islets/chip) into the cell culture chamber using a syringe and needle. Media were refreshed after the pancreatic islets attached onto the surface of the chip.

Immunofluorescence microscopy. Pancreatic ductal organoids in Matrigel were fixed with $3.7 \%$ formaldehyde for $15 \mathrm{~min}$ at room temperature and the Matrigel was broken down by pipetting with $1 \mathrm{~mL}$ EtOH. The organoids were embedded into HistoGel (Invitrogen; \#HG-4000-012) and were first examined by goldstandard morphological section and H\&E stain. Paraffin-sectioned organoids were deparaffinized for immunofluorescence microscopy. For a monolayer of PDECs on a trans-well membrane or a pancreas-on-a-chip, cells were fixed with $3.7 \%$ formaldehyde for $15 \mathrm{~min}$ at room temperature. Cells were then permeabilized using $1 \times$ permeabilization solution (eBioscience; \#00-8333-56) for $8 \mathrm{~min}$ at room temperature and washed three times with PBS for 5 min each. Cells were then blocked using 1\% goat serum (Sigma-Aldrich; \#A8806-5G) for $1 \mathrm{~h}$ at room temperature and incubated with primary antibodies (diluted in antibody diluent (Invitrogen; \#TA125-ADQ) 1:100), anti-CFTR R1104 (Eric Sorscher lab, CF Center, University of Alabama, Birmingham, AL, USA [presently, Emory University, Atlanta, GA, USA]), anti-ZO-1 (BD Biosciences; \#610967), anti-ENaC (Invitrogen; \#PA1-920A), anti-KRT 19 (Invitrogen; \#MA5-12663), anti-E cadherin (Cell Signaling Technology; \#3195), anti-insulin (Cell Signaling; \#C27C9), and anti-glucagon (Sigma; \#G2654) overnight at $4{ }^{\circ} \mathrm{C}$. Cells were washed three times with PBS for 5 min each and incubated with secondary antibodies (Invitrogen; Alexa Fluor 488 or 568; 1:500) for $1 \mathrm{~h}$ at room temperature. Alexa Fluor 488 Phalloidin (Invitrogen; \#A12379; 1:50) was employed to the secondary antibody for F-actin staining following washing three times with PBS for $5 \mathrm{~min}$ each. Cells were incubated with DAPI solution (Invitrogen; \#D1306; 1:500) for 20 min for nucleus staining and washed with PBS. For the trans-well membrane, cut edge of the membrane and transferred the membrane with cells onto a glass slide oriented cell-side up. Cells were then mounted in Vecta-shield mounting medium (Vector Labs; \#H-1000). A cover slip was placed onto the cells and fixed with nail polish. For the pancreas-ona-chip, cell culture chambers were separated manually by hands followed by nuclear staining with 4',6-diamidino-2-phenylindole (DAPI) solution for $20 \mathrm{~min}$. The porous membrane with cells remained on the upper layer. One drop of mounting solution was applied onto the cells and a coverslip was placed for imaging. Fluorescence images were obtained using a confocal microscope (Olympus FV1200). Combined images were created using an Image J software provided by NIH.

Extract RNA from pancreatic ductal organoids. Organoid growth media were discarded and the Matrigel was broken down by pipetting with $1 \mathrm{~mL}$ PBS. Pancreatic ductal organoids were picked and transferred to $1.5 \mathrm{~mL}$ RNA-free tube manually using a $200 \mu \mathrm{L}$ pipette. The supernatant and Matrigel were discarded after microcentrifuge at $16,800 \times g$ for $5 \mathrm{~min}$ and RNA was extracted using an Ambion miRNA Isolation Kit (Invitrogen; \#AM1561) using the protocol provided by Ambion.

Monitoring CFTR function. CFTR function of pancreatic ductal organoids was monitored using the fluid secretion assay ${ }^{26}$ in response to an intracellular cAMP activating agonist (FSK; $10 \mu \mathrm{M})$ for $2 \mathrm{~h}$ at $37^{\circ} \mathrm{C}$. Fluid secretion was calculated by measuring the volume ratio of luminal area over the entire organoid pre-treatment and post treatment with FSK. Fluid secretions were monitored at day 4 after isolation of organoids with at least 20 organoids. The area of lumen and outer sphere was measured using the Image J software.

Pancreatic ductal organoids were transferred, when their diameter reached 500 $\mu \mathrm{m}$, onto trans-well membranes (Corning; \#3470), 10 organoids each as previously described. The ductal epithelial cells transformed into a polarized monolayer from spheroids on the trans-well membrane within 2 weeks. Transepithelial electrical resistance was measured using epithelial volt-ohm meter (World Precision Instruments, \#EVOM and \#STX2) and the trans-well membrane was mounted in an Ussing chamber when the resistance was over $1000 \Omega / \mathrm{cm}^{2}$. Cells were bathed in Ringer's solution (mM) for apical side (pH 7.2): $0.12 \mathrm{NaCl}, 25 \mathrm{NaHCO}_{3}, 3.3$ $\mathrm{KH}_{2} \mathrm{PO}_{4}, 0.83 \mathrm{~K}_{2} \mathrm{HPO}_{4}, 1.2 \mathrm{CaCl}_{2}, 1.2 \mathrm{MgCl}_{2}, 141 \mathrm{Na}$-gluconate, and 10 mannitol, and for basolateral side ( $\mathrm{pH} 7.2$ ): $120 \mathrm{NaCl}, 25 \mathrm{NaHCO}_{3}, 3.3 \mathrm{KH}_{2} \mathrm{PO}_{4}, 0.83$ $\mathrm{K}_{2} \mathrm{HPO}_{4}, 1.2 \mathrm{CaCl}_{2}, 1.2 \mathrm{MgCl}_{2}$, and $10 \mathrm{D}$-glucose maintained the temperature of the bath using circulate system as $37^{\circ} \mathrm{C}^{26,46}$. CFTR function was monitored in real time in response to current changing by FSK. When the current showed a stable baseline, $10 \mu \mathrm{M}$ FSK was added to the apical side for CFTR channel opening. For CFTR channel closing, CFTR channel inhibitor, CFTR $_{\text {inh-172 }}(20 \mu \mathrm{M})$, was applied to the apical side.

CFTR function of PDECs was monitored using iodide efflux assay ${ }^{47}$. Cell culture media were washed out with $136 \mathrm{mM} \mathrm{NaNO}_{3}$ and incubated with $136 \mathrm{mM}$ $\mathrm{NaI}$ for $1 \mathrm{~h}$ at $37^{\circ} \mathrm{C}$. After 1-h incubation, cells were washed with $300 \mu \mathrm{L}$ of $\mathrm{NaNO}_{3}$ $(136 \mathrm{mM})$ and the supernatant was collected with $136 \mathrm{mM} \mathrm{NaNO}_{3}$ using a syringe and needle. A $1.5 \mathrm{~mL}$ tube was placed on a digital weighing scale and the supernatant was dropped into the tube with recording the weight for approximately $20 \mu \mathrm{L}$ of each sample. The first 10 samples were collected with $136 \mathrm{mM} \mathrm{NaNO}_{3}$ and the other 10 samples with $136 \mathrm{mM} \mathrm{NaNO}_{3}$ containing $10 \mu \mathrm{M}$ FSK. Iodide concentration was calculated using an electrolyte detector (Thermo 
Orion; \#420) with electrode probe filled with specific iodide-sensitive electrolyte (Invitrogen; \#900063). The electrode was immersed in $5 \mathrm{~mL}$ of $100 \mathrm{mM} \mathrm{NaNO}_{3}$ (stirred) to detect iodide. Voltage change was measured by adding each sample serially. A standard curve was obtained using $10 \mu \mathrm{M}, 100 \mu \mathrm{M}$, and $1 \mathrm{mM} \mathrm{NaI}$.

Monitoring insulin secretion. Cell culture media were discarded just before collection for the measurement. Media $(60 \mu \mathrm{L})$ were collected and incubated with refreshed media for $1 \mathrm{~h}$ at $37^{\circ} \mathrm{C}, 5 \% \mathrm{CO}_{2}$. After 1-h incubation, an additional $60 \mu \mathrm{L}$ media were collected. Collected media were placed on ice until ready to assay. For stimulation of pancreatic islets, $450 \mathrm{mg} / \mathrm{dL}$ glucose-containing media (instead of $100 \mathrm{mg} / \mathrm{dL}$ ) were used. Insulin secretion was monitored by measuring concentration of insulin in the culture media using ELISA (Invitrogen; \#KAQ1251) following a protocol provided by the company.

To monitor insulin secretion from pancreas-on-a-chip, we co-cultured PDECs in the top chamber and pancreatic islets in the bottom chamber. Base media for PDECs, advanced DMEM/F12, contains insulin, which can affect the concentration of insulin secreted by pancreatic islets in the bottom chamber. It was switched to DMEM (same as pancreatic islets media). Two chips were prepared, Chip A and Chip B, to employ agonist $\left(10 \mu \mathrm{M}\right.$ FSK) or inhibitor $\left(20 \mu \mathrm{M} \mathrm{CFTR}_{\text {inh-172 }}\right)$ of CFTR channel on the PDECs. The chips were incubated at $37^{\circ} \mathrm{C}$ for $1 \mathrm{~h}$ and $60 \mu \mathrm{L}$ media

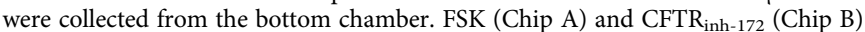
were employed on the top chambers and the chips were incubated at $37^{\circ} \mathrm{C}$ for $1 \mathrm{~h}$. Sixty microliters of media was collected from the pancreatic islets on the bottom chamber. For Chip A, the media in the bottom chamber were switched to highglucose-containing media $(450 \mathrm{mg} / \mathrm{dL})$ and the chip was incubated at $37^{\circ} \mathrm{C}$ for $1 \mathrm{~h}$. For Chip B, a combination of FSK and CFTR inh-172 $_{12}$ were added to the top chamber and the chip was incubated at $37^{\circ} \mathrm{C}$ for $1 \mathrm{~h}$. The chip was incubated with highglucose-containing media at $37^{\circ} \mathrm{C}$ for $1 \mathrm{~h}$. Sixty microliters of media were collected from the pancreatic islets in the bottom chamber.

Statistical analysis. Data were derived from at least three independent replicates. The level of marginal significance, $p$-value, was calculated using two-tailed Student's $t$ test for pairwise comparison and one-way analysis of variance with Bonferroni adjustment for multiple variations. A $p$ value $<0.05$ was considered significant.

Reporting summary. Further information on research design is available in the Nature Research Reporting Summary linked to this article.

\section{Data availability}

All experimental data relevant to graphs and images within the article and supplementary information are available from corresponding authors upon request.

Received: 11 September 2018 Accepted: 27 June 2019

Published online: 16 July 2019

\section{References}

1. Marino, C. R., Matovcik, L. M., Gorelick, F. S. \& Cohn, J. A. Localization of the cystic fibrosis transmembrane conductance regulator in pancreas. J. Clin. Invest. 88, 712-716 (1991).

2. Zielenski, J. Genotype and phenotype in cystic fibrosis. Respiriration 67, 117-133 (2000).

3. Sheppard, D. N. et al. Mutations in CFTR associated with mild-disease-form $\mathrm{Cl}^{-}$channels with altered pore properties. Nature 362, 160-164 (1993).

4. O’Sullivan, B. P. \& Freedman, S. D. Cystic fibrosis. Lancet 373, 1891-1904 (2009).

5. Andersen, D. H. Cystic fibrosis of the pancreas and its relation to celiac disease: a clinical and pathologic study. Am. J. Dis. Child. 56, 344-399 (1938).

6. Harutyunyan, M. et al. Personalized medicine in CF: from modulator development to therapy for cystic fibrosis patients with rare CFTR mutations. Am. J. Physiol. Lung Cell. Mol. Physiol. 314, 1529-1543 (2018).

7. Moran, A. et al. Cystic fibrosis-related diabetes: current trends in prevalence, incidence, and mortality. Diabetes Care 32, 1626-1631 (2009).

8. Lanng, S. Glucose intolerance in cystic fibrosis patients. Paediatr. Respir. Rev. 2, 253-259 (2001).

9. Konrad, K. et al. Cystic fibrosis-related diabetes compared with type 1 and type 2 diabetes in adults. Diabetes Metab. Res. Rev. 29, 568-575 (2013).

10. Marshall, B. C. et al. Epidemiology of cystic fibrosis-related diabetes. J. Pediatr. 146, 681-687 (2005).

11. Saint-Criq, V. \& Gray, M. A. Role of CFTR in epithelial physiology. Cell Mol. Life Sci. 74, 93-115 (2017).

12. Strong, T. V., Boehm, K. \& Collins, F. S. Localization of cystic fibrosis transmembrane conductance regulator mRNA in the human gastrointestinal tract by in situ hybridization. J. Clin. Invest. 93, 347-354 (1994).
13. Wilschanski, M. \& Novak, I. The cystic fibrosis of exocrine pancreas. Cold Spring Harb. Perspect. Med. 3, https://doi.org/10.1101/cshperspect.a009746 (2013).

14. Efrat, S. \& Russ, H. A. Making $\beta$ cells from adult tissues. Trends Endocrinol. Metab 23, 278-285 (2012).

15. Bellin, M. D. et al. Total pancreatectomy and islet autotransplantation in chronic pancreatitis: recommendations from PancreasFest. Pancreatology 14, 27-35 (2014).

16. Terry, S. C., Jerman, J. J. \& Anyell., J. B. A gas chromatographic air analyzer fabricated on a silicon wafer. IEEE Trans. Electron. Dev. 26 1880-1886 (1979).

17. Golden, A. P. \& Tien, J. Fabrication of microfluidic hydrogels using molded gelatin as a sacrificial element. Lab Chip 7, 720-725 (2007).

18. Sung, K. E. et al. Control of 3-dimensional collagen matrix polymerization for reproducible human mammary fibroblast cell culture in microfluidic devices. Biomaterials 30, 4833-4841 (2009).

19. Vickerman, V., Blundo, J., Chung, S. \& Kamm, R. Design, fabrication and implementation of a novel multi-parameter control microfluidic platform for three-dimensional cell culture and real-time imaging. Lab Chip 8, 1468-1477 (2008).

20. Sanchez-Freire, V., Ebert, A. D., Kalisky, T., Quake, S. R. \& Wu, J. C. Microfluidic single-cell real-time PCR for comparative analysis of gene expression patterns. Nat. Protoc. 7, 829-838 (2012).

21. Kang, G., Ward, T. M., Bockhorn, J., Pegram, M. D. \& Herr A. E. HER2 protein isoform heterogeneity investigated by single-cell western blotting. Cancer Res. 76, https://doi.org/10.1158/1538-7445.AM2016-352 (2016).

22. Koh, A. et al. A soft, wearable microfluidic device for the capture, storage, and colorimetric sensing of sweat. Sci. Transl. Med. 8, 366ral65 (2016).

23. Huh, D. et al. Microfabrication of human organs-on-chips. Nat. Protoc. 8, 2135-2157 (2013).

24. Bhatia, S. N. \& Ingber, D. E. Microfluidic organs-on-chips. Nat. Biotechnol. 32, 760-772 (2014).

25. Hansen, W. A. et al. Supravital dithizone staining in the isolation of human and rat pancreatic-islets. Diabetes Res. 10, 53-57 (1989).

26. Moon, C. et al. Compartmentalized accumulation of cAMP near complexes of multidrug resistance protein 4 (MRP4) and cystic fibrosis transmembrane conductance regulator (CFTR) contributes to drug-induced diarrhea. J. Biol. Chem. 290, 11246-11257 (2015).

27. Rock, J. R. et al. Basal cells as stem cells of the mouse trachea and human airway epithelium. Proc. Natl. Acad. Sci. USA 106, 12771 (2009).

28. Broutier, L. et al. Culture and establishment of self-renewing human and mouse adult liver and pancreas 3D organoids and their genetic manipulation. Nat. Protoc. 11, 1724-1743 (2016).

29. Mahe, M. M., Sundaram, N., Watson, C. L., Shroyer, N. F. \& Helmrath, M. A Establishment of human epithelial enteroids and colonoids from whole tissue and biopsy. J. Vis. Exp. https://doi.org/10.3791/52483 (2015).

30. Ingber, D. E. Developmentally inspired human "organs on chips". Development 145, https://doi.org/10.1242/dev.156125 (2018).

31. Maleth, J. et al. Alcohol disrupts levels and function of the cystic fibrosis transmembrane conductance regulator to promote development of pancreatitis. Gastroenterology 148, 427-439 e416 (2015).

32. Toepke, M. W. \& Beebe, D. J. PDMS absorption of small molecules and consequences in microfluidic applications. Lab Chip 6, 1484-1486 (2006).

33. van Meer, B. J. et al. Small molecule absorption by PDMS in the context of drug response bioassays. Biochem. Biophys. Res. Commun. 482, 323-328 (2017).

34. Tan, S. H., Nguyen, N. T., Chua, Y. C. \& Kang, T. G. Oxygen plasma treatment for reducing hydrophobicity of a sealed polydimethylsiloxane microchannel. Biomicrofluidics 4, 32204 (2010).

35. Bodas, D. \& Khan-Malek, C. Hydrophilization and hydrophobic recovery of PDMS by oxygen plasma and chemical treatment-An SEM investigation. Sens. Actuators B 123, 368-373 (2007).

36. Recek, N. et al. Adsorption of proteins and cell adhesion to plasma treated polymer substrates. Int. J. Polym. Mater. Polym. Biomater. 63, 685-691 (2014).

37. Muck, A. et al. Fabrication of oly(methyl methacrylate) microfluidic chips by atmospheric molding. Anal. Chem. 76, 2290-2297 (2004).

38. Do, J. et al. Development of functional lab-on-a-chip on polymer for point-ofcare testing of metabolic parameters. Lab Chip 8, 2113-2120 (2008).

39. Domansky, K. et al. SEBS elastomers for fabrication of microfluidic devices with reduced drug absorption by injection molding and extrusion. Microfluid. Nanofluidics 21, 107 (2017).

40. Roman, G. T., Hlaus, T., Bass, K. J., Seelhammer, T. G. \& Culbertson, C. T. Sol-gel modified poly(dimethylsiloxane) microfluidic devices with high electroosmotic mobilities and hydrophilic channel wall characteristics. Anal. Chem. 77, 1414-1422 (2005).

41. Ostuni, E., Chen, C. S., Ingber, D. E. \& Whitesides, G. M. Selective deposition of proteins and cells in arrays of microwells. Langmuir 17, 2828-2834 (2001). 
42. Kelly, O. G. et al. Cell-surface markers for the isolation of pancreatic cell types derived from human embryonic stem cells. Nat. Biotechnol. 29, 750-756 (2011).

43. Watanabe, K. et al. A ROCK inhibitor permits survival of dissociated human embryonic stem cells. Nat. Biotechnol. 25, 681-686 (2007).

44. Schneider, F., Draheirn, J., Kamberger, R. \& Wallrabe, U. Process and material properties of polydimethylsiloxane (PDMS) for optical MEMS. Sens. Actuators A 151, 95-99 (2009).

45. Vannoort, R. \& Bayston, R. Mechanical-properties of antibacterial siliconerubber for hydrocephalus shunts. J. Biomed. Mater. Res. 13, 623-630 (1979).

46. Li, C. et al. Spatiotemporal coupling of cAMP transporter to CFTR chloride channel function in the gut epithelia. Cell 131, 940-951 (2007).

47. Arora, K. et al. Stabilizing rescued surface-localized deltaf508 CFTR by potentiation of its interaction with $\mathrm{Na}(+) / \mathrm{H}(+)$ exchanger regulatory factor 1 . Biochemistry 53, 4169-4179 (2014).

\section{Acknowledgements}

We thank Dr. Gail Pyne-Geithman for editing the manuscript. This research was supported by the National Institutes of Health (DK080834, DK093045, P30 DK117467), and CF Foundation grants to K.S.M. (MUN18F0) and A.P.N. (NAREN14XX0).

\section{Author contributions}

K.S.M. performed all experiments and was assisted by K.A. (imaging, RNA-sequencing, and experimental design), Y.H. (experimental design),), F.Y. (RNA-sequencing), Y.R. (imaging), and B.N.A. (culturing pancreatic islets in vitro). K.S.M. fabricated the microfluidic device assisted by S.Y., J.D.N., and M.A.-E.-H., and J.J.P. performed the surgery, TPIAT. K.S.M., K.A., Y.H., M.A.-E.-H., J.D.N. J.J.P, and A.P.N wrote and edited the manuscript. A.P.N., J.D.N., and J.J.P. designed the project.

\section{Additional information}

Supplementary Information accompanies this paper at https://doi.org/10.1038/s41467019-11178-w.

Competing interests: The authors declare no competing interests.

Reprints and permission information is available online at http://npg.nature.com/ reprintsandpermissions/

Peer review information: Nature Communications thanks the anonymous reviewer(s) for their contribution to the peer review of this work. Peer reviewer reports are available.

Publisher's note: Springer Nature remains neutral with regard to jurisdictional claims in published maps and institutional affiliations.

(c) (i) Open Access This article is licensed under a Creative Commons Attribution 4.0 International License, which permits use, sharing, adaptation, distribution and reproduction in any medium or format, as long as you give appropriate credit to the original author(s) and the source, provide a link to the Creative Commons license, and indicate if changes were made. The images or other third party material in this article are included in the article's Creative Commons license, unless indicated otherwise in a credit line to the material. If material is not included in the article's Creative Commons license and your intended use is not permitted by statutory regulation or exceeds the permitted use, you will need to obtain permission directly from the copyright holder. To view a copy of this license, visit http://creativecommons.org/ licenses/by/4.0/.

(C) The Author(s) 2019 